\title{
Improved electrical and mechanical properties for the reduced graphene oxide decorated polymer nanofiber composite with a core shell structure
}

Nan Zheng ${ }^{1}$, Yang Song ${ }^{1}$, Ling Wang 2 , Jiefeng Gao ${ }^{* 2,3}$, Yu Wang ${ }^{1}$, Xiaoli Dong ${ }^{* 1}$

1. School of Light Industry and Chemical Engineering, Dalian Polytechnic University, Dalian, 116034, P. R. China

2. College of Chemistry and Chemical Engineering, Yangzhou University, Yangzhou, Jiangsu, 225002, P. R. China

3. State Key Laboratory of Polymer Materials Engineering Sichuan University,

Chengdu, Sichuan 610065, P. R. China

Corresponding authors: ifgao@yzu.edu.cn (Jiefeng Gao); dongxiaoli65@163.com (Xiaoli Dong)
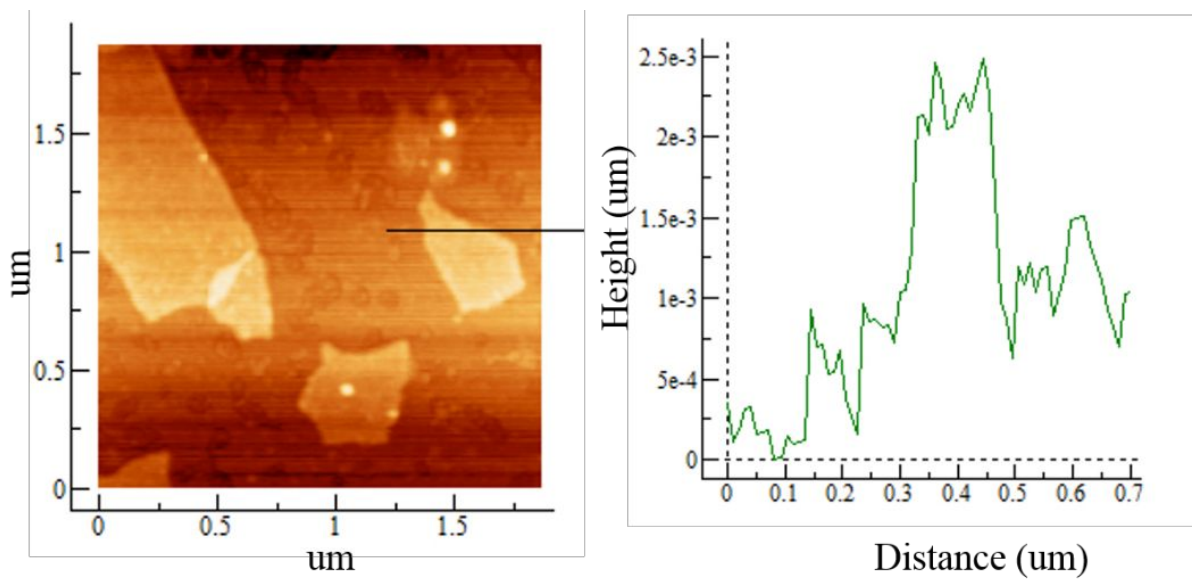

Figure S1 AFM images and corresponding height profiles of RGO 


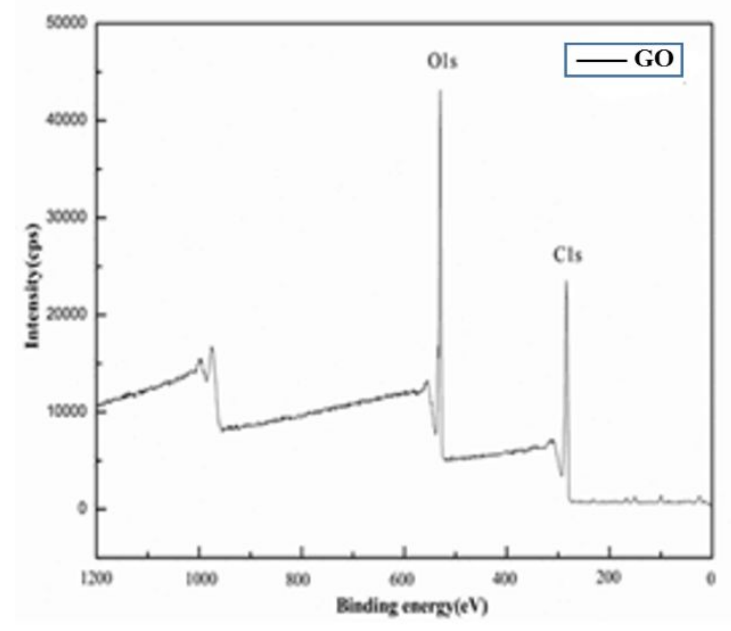

(a)

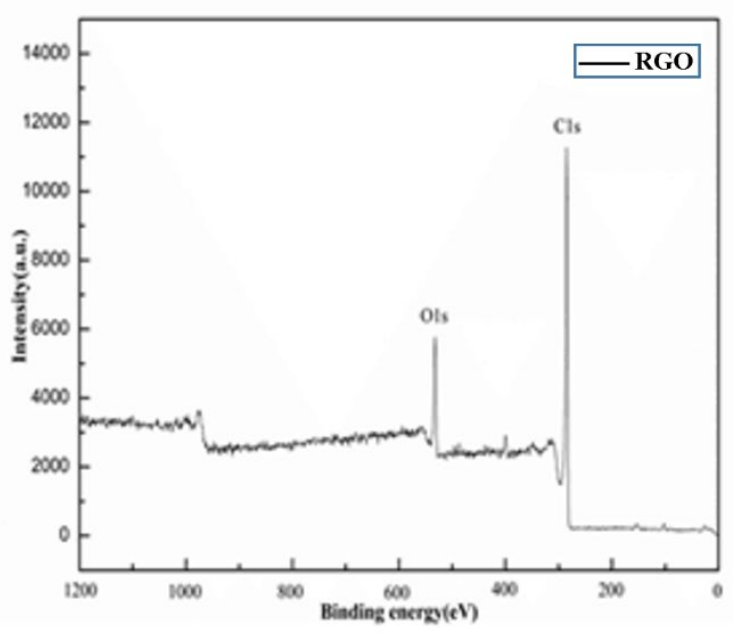

(b)

Figure S2 XPS results for (a) GO and (b) RGO

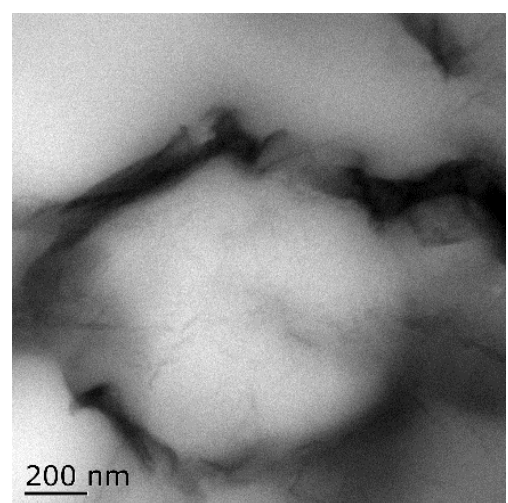

Figure S3 TEM image of cross-section of the nanofiber composite possessing a polymer nanofiber core/graphene RGO. 